\title{
Photoelectrochemical Water Splitting System-A Study of Interfacial Charge Transfer with Scanning Electrochemical Microscopy
}

\author{
Bingyan Zhang ${ }^{\S}$, Xiaofan Zhang ${ }^{\S}$, Xin Xiao, Yan Shen* \\ Wuhan National Laboratory for Optoelectronics, School of Optoelectronic Science and Engineering, Huazhong University \\ of Science and Technology, Wuhan 430074, P. R. China. \\ Corresponding Author: Yan Shen*, E-mail: ciac_sheny@mail.hust.edu.cn
}

Table of contents:

SI-1 Experimental section

SI-2 UV-visible absorption spectroscopy measurement

SI-3 Morphology characterization for the samples

SI-4 Detailed description of the feedback mode of SECM

SI-5 Complete derivation of SECM model for photocatalyst regeneration $\quad$ S6

SI-6 SECM measurements for interfacial reaction kinetics in photoelectrochemical water splitting $\quad$ S9

SI-6.1 Normalized approach curves towards $\mathrm{FTO} / \mathrm{BiVO}_{4}$ film in dark and under blue illumination $\quad$ S9

SI-6.2 Normalized approach curves towards FTO/Mo: $\mathrm{BiVO}_{4}$ film under dark $\quad$ S10

SI-6.3 Normalized approach curves towards FTO/Mo: $\mathrm{BiVO}_{4}$ film under blue illumination $\quad$ S11

SI-6.4 Calculated data from SECM measurement $\quad$ S11

SI-7 X-ray photoelectron spectroscopy measurement $\quad$ S16

$\begin{array}{ll}\text { References } & \text { S16 }\end{array}$ 


\section{SI-1 Experimental section}

The synthetic scheme is similar to the method that reported by the previous literatures. ${ }^{1,2}$ Firstly, fluorine-doped tin oxide (FTO, sheet resistance $\sim 15 \Omega$ ) was used as the working electrode, Pt wire as counter electrode, and $\mathrm{Ag} / \mathrm{AgCl}(3 \mathrm{M} \mathrm{KCl})$ was as reference electrode. The plating solution was prepared by dissolving $40 \mathrm{mM} \mathrm{Bi}\left(\mathrm{NO}_{3}\right)_{3}$ in a $400 \mathrm{mM} \mathrm{KI}$ aqueous solution. The $\mathrm{pH}$ of the resulting opaque orange solution was adjusted to 1.7 by using dilute $\mathrm{HNO}_{3}$. Then $50 \mathrm{mM} \mathrm{p}$-benzoquinone was added to the solution, resulting in a black solution. The depositions were carried out potentiostatically at $-0.1 \mathrm{~V}$ (vs. $\mathrm{Ag} / \mathrm{AgCl}$ ) for 5 min under room temperature. After the deposition, the electrode was rinsed with deionized water and dried with a gentle stream of nitrogen gas. Secondly, synthesis of $\mathrm{BiVO}_{4}$ electrode, $0.1 \mathrm{~mL}$ of a dimethyl sulfoxide (DMSO) solution containing 0.2 M vanadyl acetylacetonate ( $\left.\mathrm{VO}(\mathrm{acac})_{2}\right)$ was evenly coated at the surface of the $\mathrm{BiOI}$ electrode. Then the prepared electrode was heated in a muffle furnace at $450^{\circ} \mathrm{C}$ under air atmosphere for $2 \mathrm{~h}$ to convert $\mathrm{BiOI}$ to $\mathrm{BiVO}_{4}$. Thirdly, synthesis of $\mathrm{Mo}: \mathrm{BiVO}_{4}$ electrode, $0.1 \mathrm{~mL}$ of a dimethyl sulfoxide solution containing $0.2 \mathrm{M}$ vanadyl acetylacetonate $\left(\mathrm{VO}(\mathrm{acac})_{2}\right)$ and Mo powder $(0.02 \mathrm{M})$ was placed on the BiOI electrode and was heated in a muffle furnace at $450{ }^{\circ} \mathrm{C}$ under air atmosphere for $2 \mathrm{~h}$ in air to convert $\mathrm{BiOI}$ to $\mathrm{Mo}-\mathrm{BiVO}_{4}$. The excess $\mathrm{V}_{2} \mathrm{O}_{5}$ presents in the surface of $\mathrm{BiVO}_{4}$ or $\mathrm{Mo}: \mathrm{BiVO}_{4}$ electrode was removed by soaking them in $1 \mathrm{M} \mathrm{KOH}$ solution for 30 min with gentle stirring. The resulting pristine $\mathrm{BiVO}_{4}$ or $\mathrm{Mo}: \mathrm{BiVO}_{4}$ electrodes were rinsed again with DI water and dried with a gentle stream of nitrogen gas. 


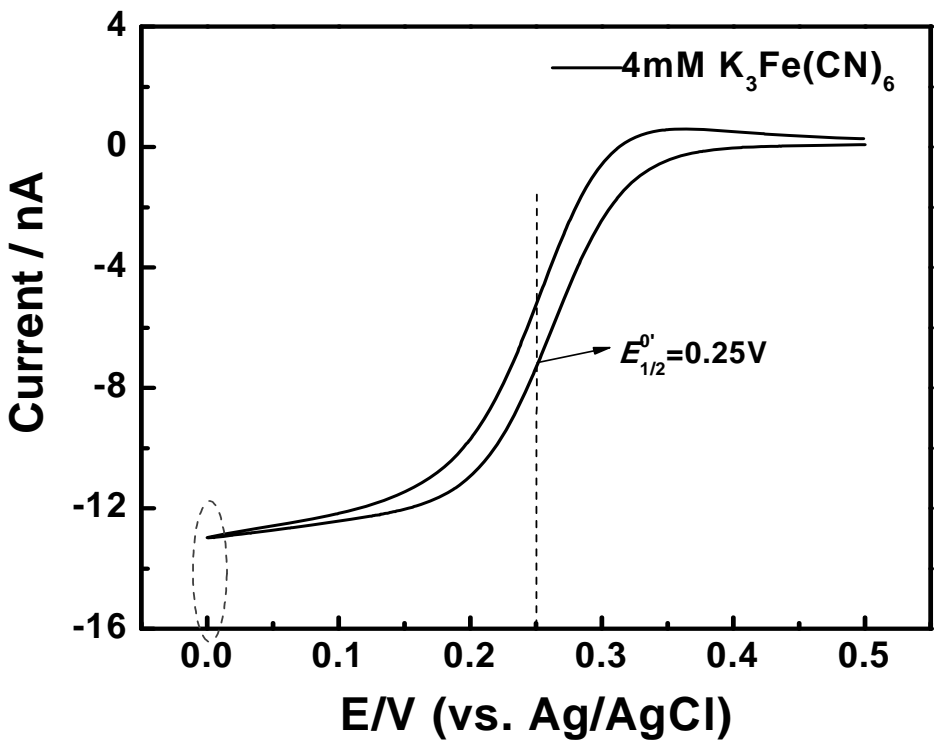

Figure S1. Cyclic voltammetric response of Pt ultramicroelectrode towards $4 \mathrm{mM}\left[\mathrm{Fe}(\mathrm{CN})_{6}\right]^{3-}$ at a scan rate of $50 \mathrm{mV} \mathrm{s}^{-1}$, in $0.1 \mathrm{M}$ PBS $(\mathrm{pH}=7.0)+0.5 \mathrm{M} \mathrm{Na}_{2} \mathrm{SO}_{4}$.

SI-2 UV-visible absorption spectroscopy measurement

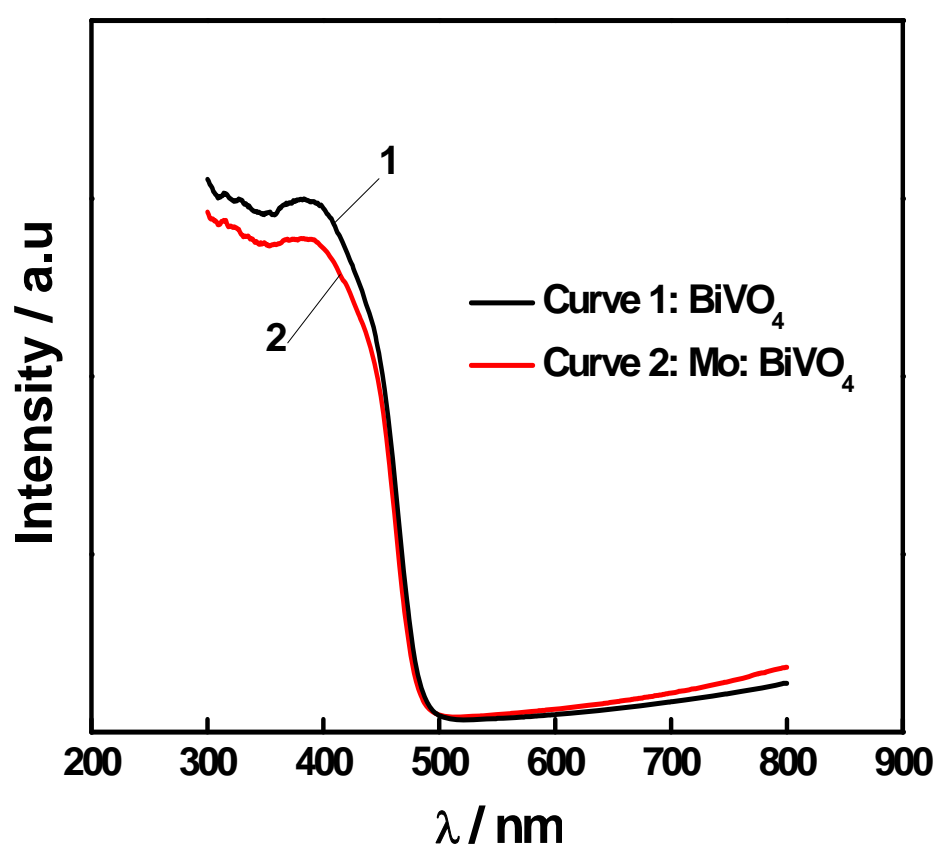

Figure S2. UV-visible absorption spectroscopy of the $\mathrm{FTO} / \mathrm{BiVO}_{4}$ and $\mathrm{FTO} / \mathrm{Mo}: \mathrm{BiVO}_{4}$ samples. 

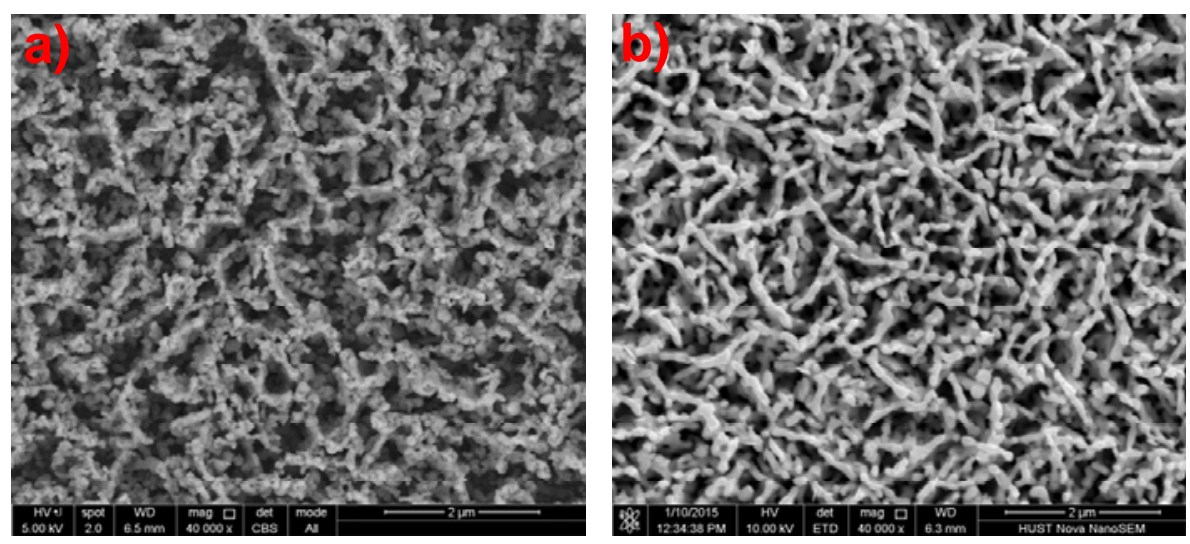

Figure S3. Typical SEM images of (a) $\mathrm{BiVO}_{4}$, and (b) Mo:BiVO 4 .

\section{SI-4 Detailed description of SECM with feedback mode}

In this study SECM was employed to scrutinize interfacial reaction kinetics in photoelectrochemical water splitting with a feedback mode at the $\mathrm{BiVO}_{4} /$ electrolyte heterojunction through the reduction of the oxidized photoelectric catalyst cations. Figure S4-S6 presents the approaching curves (tip current-distance relationships) for the case of $\mathrm{FTO} / \mathrm{BiVO}_{4}$ and $\mathrm{FTO} / \mathrm{Mo}: \mathrm{BiVO}_{4}$ in the electrolyte containing $\left[\mathrm{Fe}(\mathrm{CN})_{6}\right]^{3-}$ $/\left[\mathrm{Fe}(\mathrm{CN})_{6}\right]^{4-}$ as redox probe under dark or illumination.

The theory, methodology, and instrumentation in SECM have been reviewed in reference 3 . The basic concept of interest in connection with measurements at the photoanode/electrolyte is that of the tip feedback current. When the ultramicroelectrode tip is far from any surface, the tip current, $\mathrm{i}_{\mathrm{T}, \infty}$ is determined by the number of electrons involved in the electrode reaction, the diffusion coefficient and the concentration of the electroactive reactants, and the radius of the ultramicroelectrode. When the tip is close to a surface, the tip current, $\mathrm{i}_{\mathrm{T}}$, is perturbed by blockage of diffusion of Ox to the tip by the substrate (assumption of reduction reaction onto the tip) and by reactions that occur at the substrate surface. If the substrate surface is an insulator or a surface where the tip generated product, Red, does not react, $\mathrm{i}_{\mathrm{T}}<\mathrm{i}_{\mathrm{T}, \infty}$. This is termed negative feedback. If the oxidation of Red to Ox occurs at the substrate, the current will be larger than that seen in the negative feedback approach curve, depending on the rate of the reaction. For sufficiently high rate constants, $\mathrm{k}$, for this oxidation reaction on the substrate, $\mathrm{i}_{\mathrm{T}}$ must be greater than $\mathrm{i}_{\mathrm{T}, \infty}$ (positive feedback). Typical approach curves as a function of $d$ are shown in Figure 3a. By comparing an 
experimental approach curve to theoretical ones, the rate constant for a reaction at the substrate can be obtained.

In this study, during the SECM measurement with feedback model, the tip current is determined by the reduction of $\mathrm{Ox}$ in the solution in dark (the substrate can be considered as insulating electrode, i.e., negative feedback). However, when light is shed onto the electrode, an active electrode, the Red generated by the tip can be converted to oxidation state again via reactions on the active substrate electrode/electrolyte interface (the substrate can be considered as conducting electrode, i.e., positive feedback). Therefore, the feedback current is from the conversion of the mediator in the solution. ${ }^{3}$

Thus in this study, there will be only one form of the redox mediator added in solution. Clearly, smaller concentration of the mediator provided in the solution, the difference between the feedback current and the base current would be easy to determine. According to the Equations S1-6, the first order kinetics constant $k_{\text {eff }}$ can be depended on the concentration of the corresponding species to some extent.

The normalized approach curve can be described with following equations: ${ }^{4}$

$$
\begin{gathered}
I_{T}(L, \kappa, R G)=I_{T}^{\text {cond }}\left(L+\frac{1}{\kappa}, R G\right)+\frac{I_{T}^{\text {ins }}(L, R G)-1}{\left(1+2.47 R G^{0.31} L k\right)\left(1+L^{0.006 R G+0.1} 13 k^{-0.023 R G+0.91}\right)} \\
I_{T}^{\text {ins }}(L, R G)=\frac{\left(2.08 / R G^{0.358}\right)(L-(0.145 / R G))+1.585}{\left(2.08 / R G^{0.358}\right)(L+0.00238 R G)+1.57+(\ln R G / L)+(2 / \pi R G) \ln (1+(\pi R G / 2 L))} \\
I_{T}^{\text {cond }}\left(L+\kappa^{-1}, R G\right)=\alpha(R G)+\frac{\pi}{4 \beta(R G) \arctan \left(L+\kappa^{-1}\right)}+\left(1-\alpha(R G)-\frac{1}{2 \beta(R G)}\right) \frac{2}{\pi} \arctan \left(L+\kappa^{-1}\right) \\
\alpha(R G)=\ln 2+\ln 2\left(1-\frac{2}{\pi} \arccos \left(\frac{1}{R G}\right)\right)-\ln 2\left(1-\left(\frac{2}{\pi} \arccos \left(\frac{1}{R G}\right)\right)^{2}\right) \quad(S-5) \\
\beta(R G)=1+0.639\left(1-\frac{2}{\pi} \arccos \left(\frac{1}{R G}\right)\right)-0.186\left(1-\left(\frac{2}{\pi} \arccos \left(\frac{1}{R G}\right)\right)^{\text {ins }}\right) \quad(S-6)
\end{gathered}
$$

where $\mathrm{RG}=r_{\text {glass }} / r_{\mathrm{T}}$ is the ratio of the radius of the glass sheath $\left(r_{\text {glass }}\right)$ to the radius of the active area of the Pt UME $\left(r_{\mathrm{T}}\right), I_{T}^{\text {cond }}$ is the diffusion controlled mediator recycling at the sample ("positive feedback") and $I_{T}^{\text {ins }}$ describes the current if no reaction occurs at the sample (insulating sample, "negative feedback"). In Equations S1-S5, the important apparent heterogeneous electron transfer rate constant $\kappa$ can be obtained by fitting the experimental approach curves with the known parameters, including the RG, $L, r_{\mathrm{T}}$, etc. 


\section{SI-5 complete derivation of SECM model for photocatalyst regeneration}

The following reaction mechanism for photocatalyst regeneration was developed under steady state SECM experiments for the dynamic equilibrium process of the charge transfer with $\left[\mathrm{Fe}(\mathrm{CN})_{6}\right]^{3-}$ $/\left[\mathrm{Fe}(\mathrm{CN})_{6}\right]^{4-}$ as redox probe. ${ }^{5}$

$$
\begin{aligned}
& \mathrm{BiVO}_{4} / \mathrm{FTO}+\mathrm{hv} \stackrel{\phi_{h v}}{\longrightarrow} \mathrm{BiVO}_{4} * / \mathrm{FTO} \\
& \mathrm{BiVO}_{4} * / \mathrm{FTO} \stackrel{k_{i j j}}{\longrightarrow} \mathrm{BiVO}_{4}^{+} / \mathrm{FTO}+e_{\mathrm{CB}}^{-}(\mathrm{FTO}) \\
& \mathrm{BiVO}_{4}^{+} / \mathrm{FTO}+\left[\mathrm{Fe}(\mathrm{CN})_{6}\right]^{4-} \stackrel{k_{1}}{\longrightarrow} \mathrm{BiVO}_{4} / \mathrm{FTO}+\left[\mathrm{Fe}(\mathrm{CN})_{6}\right]^{3-} \\
& {\left[\mathrm{Fe}(\mathrm{CN})_{6}\right]^{3-}+e^{-} \stackrel{\mathrm{Tip}}{\longrightarrow}\left[\mathrm{Fe}(\mathrm{CN})_{6}\right]^{4-}}
\end{aligned}
$$

Steady state for $\left[\mathrm{BiVO}_{4}{ }^{*}\right]$,

$$
\begin{aligned}
& \frac{\partial\left[\mathrm{BiVO}_{4}{ }^{*}\right]}{\partial t}=0=\phi_{h v} J_{h v}\left[\mathrm{BiVO}_{4}\right]-k_{i n j}\left[\mathrm{BiVO}_{4}{ }^{*}\right] \\
& \frac{\left[\mathrm{BiVO}_{4}\right]}{\left[\mathrm{BiVO}_{4}{ }^{*}\right]}=\frac{k_{i n j}}{\phi_{h v} J_{h v}}
\end{aligned}
$$

And diffusion limited tip current $(\mathrm{n}=1)$ for reduction of $\left[\mathrm{Fe}(\mathrm{CN})_{6}\right]^{3-}$ :

$$
\begin{aligned}
& {\left[\mathrm{Fe}(\mathrm{CN})_{6}\right]^{3-}+e^{-} \rightarrow\left[\mathrm{Fe}(\mathrm{CN})_{6}\right]^{4-}} \\
& i_{T, \lim }=4 \mathrm{FD}_{\text {diffusion }}[\mathrm{C}]_{\left[\mathrm{Fe}(\mathrm{CN})_{6}\right]^{3-}} r_{T} I_{T}(L)
\end{aligned}
$$

Here, $\mathrm{K}_{3} \mathrm{Fe}(\mathrm{CN})_{6} / \mathrm{K}_{4} \mathrm{Fe}(\mathrm{CN})_{6}$ were coded as $\mathrm{Fe}^{3+} / \mathrm{Fe}^{2+}$ for short. Considering the steady state for $\left[\mathrm{BiVO}_{4}{ }^{+}\right.$,

$$
\begin{aligned}
& \frac{\partial\left[\mathrm{BiVO}_{4}^{+}\right]}{\partial t}=0=k_{i n j}\left[\mathrm{BiVO}_{4}{ }^{*}\right]-k_{1}\left[\mathrm{BiVO}_{4}^{+}\right]\left[\mathrm{Fe}^{2+}\right]_{S} \\
& \frac{\left[\mathrm{BiVO}_{4}^{+}\right]}{\left[\mathrm{BiVO}_{4}{ }^{*}\right]}=\frac{k_{i n j}}{k_{1}\left[\mathrm{Fe}^{2+}\right]_{S}}
\end{aligned}
$$

Mass balance for the total $\mathrm{BiVO}_{4}$ content,

$$
\left[\mathrm{BiVO}_{4}{ }^{0}\right]=\left[\mathrm{BiVO}_{4}\right]+\left[\mathrm{BiVO}_{4}^{+}\right]+\left[\mathrm{BiVO}_{4}{ }^{*}\right]=\left[\mathrm{BiVO}_{4}{ }^{*}\right]\left(\frac{\left[\mathrm{BiVO}_{4}\right]}{\left[\mathrm{BiVO}_{4}{ }^{*}\right]}+\frac{\left[\mathrm{BiVO}_{4}^{+}\right]}{\left[\mathrm{BiVO}_{4}{ }^{*}\right]}+1\right)
$$

Steady state expression for ratio of $\left[\mathrm{BiVO}_{4}{ }^{0}\right]$,

$$
\begin{aligned}
{\left[\mathrm{BiVO}_{4}{ }^{0}\right] } & =\left[\mathrm{BiVO}_{4}{ }^{*}\right]\left(\frac{k_{i n j}}{\phi_{h v} J_{h v}}+\frac{k_{i n j}}{k_{1}\left[\mathrm{Fe}^{2+}\right]_{S}}+1\right) \\
{\left[\mathrm{BiVO}_{4}{ }^{*}\right] } & =\frac{\left[\mathrm{BiVO}{ }_{4}^{0}\right]}{\frac{k_{i n j}}{\phi_{h v} J_{h v}}+\frac{k_{i n j}}{k_{1}\left[\mathrm{Fe}^{2+}\right]_{S}}+1}
\end{aligned}
$$

Expression for current $i_{k}\left[\mathrm{Fe}^{2+}\right]_{\mathrm{S}}$ is the $\mathrm{Fe}^{2+}$ concentration at the surface of the $\mathrm{BiVO}_{4} / \mathrm{FTO}$ electrode), 


$$
i_{k}=n F A\left(k_{1} l\left[\mathrm{BiVO}_{4}^{+}\right]\left[\mathrm{Fe}^{2+}\right]_{S}\right)
$$

Substitution of the bracketed term using the Bodenstein principle for the steady state experiment, ${ }^{6}$

$$
\begin{aligned}
& \left.k_{i n j}\left[\mathrm{BiVO}_{4}{ }^{*}\right]=k_{1}\left[\mathrm{BiVO}_{4}^{+}\right]\left[\mathrm{Fe}^{2+}\right]_{S}\right)(n=1) \\
& i_{k}=F A k_{i n j} l\left[\mathrm{BiVO}_{4}{ }^{*}\right]
\end{aligned}
$$

Substitute the expression for $\left[\mathrm{BiVO}_{4}{ }^{*}\right]$ from mass balance,

$$
\begin{aligned}
& i_{k}=F A k_{i n j} l \frac{\left[\mathrm{BiVO}_{4}{ }^{0}\right]}{\frac{k_{i n j}}{\phi_{h v} J_{h v}}+\frac{k_{i n j}}{k_{1}\left[\mathrm{Fe}^{2+}\right]_{S}}+1} \\
& i_{k}=\operatorname{FAl}\left[\mathrm{BiVO}_{4}{ }^{0}\right] \frac{1}{\frac{1}{\phi_{h v} J_{h v}}+\frac{1}{k_{1}\left[F e^{2+}\right]_{S}}+\frac{1}{k_{i n j}}} \\
& \frac{1}{i_{k}}=\frac{1}{F A l\left[\mathrm{BiVO}_{4}{ }^{0}\right] \phi_{h v} J_{h v}}+\frac{1}{F A l\left[\mathrm{BiVO}_{4}{ }^{0}\right] k_{1}\left[\mathrm{Fe}^{2+}\right]_{S}}+\frac{1}{F A l\left[\mathrm{BiVO}_{4}^{0}\right] k_{i n j}}
\end{aligned}
$$

Simplifying the expression for light absorption, electron injection and photocatalyst regeneration by summarizing the steps using $\left(k_{h v, e f f}\right)^{-1}=\left(k_{i n j}\right)^{-1}+\left(\phi_{h v} J_{h v}\right)^{-1} \approx\left(\phi_{h v} J_{h v}\right)^{-1}$ and $\left(k_{o x}^{\prime}\right)^{-1}=\left(k_{1}\right)^{-1}$.

$$
\begin{aligned}
& \frac{1}{i_{k}}=\frac{1}{F A l\left[\mathrm{BiVO}_{4}^{0}\right] k_{h v, e f f}}+\frac{1}{\mathrm{FAl}\left[\mathrm{BiVO}_{4}^{0}\right] k_{o x}^{\prime}\left[\mathrm{Fe}^{2+}\right]_{S}} \\
& \frac{1}{i_{k}}=\frac{1}{F A l\left[\mathrm{BiVO}_{4}^{0}\right] \phi_{h v} J_{h v}}+\frac{1}{\mathrm{FAl}\left[\mathrm{BiVO}_{4}^{0}\right] k_{o x}^{\prime}\left[\mathrm{Fe}^{2+}\right]_{S}}
\end{aligned}
$$

Therefore, the limiting substrate current would be reached if the $\mathrm{Fe}^{2+}$ concentration is the same as the $\mathrm{Fe}^{3+}$ concentration, i.e. all $\mathrm{Fe}^{2+}$ formed at the tip is available to the sample without any dilution, $\left[\mathrm{Fe}^{2+}\right]_{\mathrm{s}}=$ $\left[\mathrm{Fe}^{3+}\right]^{*}$

$$
\frac{1}{i_{k}}=\frac{1}{F A l\left[B i V O_{4}^{0}\right] \phi_{h v} J_{h v}}+\frac{1}{F A l\left[\mathrm{BiVO}_{4}^{0}\right] k_{o x}^{\prime}\left[\mathrm{Fe}^{3+}\right]^{*}}
$$

As mentioned above, the mediator that participates in the reaction occurs at the interface of electrolyte/substrate electrode is generated by the UME tip. Therefore, the effective area (A) of active substrate electrode is large enough to collect almost all mediators from the tip because the substrate is massively larger than the area of probe $\left(\pi r_{\mathrm{T}}^{2}\right)$. As shown in Figures 3, 4, S4, S5 and S6, the largest distance (L) between the tip and the substrate electrode only equals to four times of the radius $\left(4 r_{\mathrm{T}}\right)$ or two times of the diameters $(2 d)$ for the tip. Therefore, within a small distance $(\mathrm{L}<2 \mathrm{~d})$, the effective area (A) of active 
substrate electrode can be simplified to $\pi r_{\mathrm{T}}^{2}$. Therefore, normalizing the limiting substrate current by $i_{\mathrm{T}, \infty}$ yields $I_{\mathrm{k}, \mathrm{lim}}$

$$
\begin{aligned}
& I_{k, \lim }=\frac{i_{k, \lim }}{i_{T, \infty}}=\frac{i_{k, \lim }}{4 n F D\left[F e^{3+}\right]^{*} r_{T}}, \mathrm{n}=1 \text { at the tip for } \mathrm{Fe}^{3+}, \mathrm{A} \approx \pi \mathrm{r}_{\mathrm{T}}^{2} \\
& \frac{1}{I_{S}}=\frac{i_{T, \infty}}{i_{k, \lim }}+\frac{1}{I_{T, \text { cond }}}+\frac{1}{I_{e l, \lim }}, \frac{1}{I_{e l, \lim }}=0 \\
& \frac{1}{I_{S}}=\frac{1}{I_{T, \text { cond }}}+\frac{4 F D_{\text {diffusion }}\left[F e^{3+}\right]^{*} r_{T}}{F \pi r_{T}^{2} l\left[\mathrm{BiVO}_{4}^{0}\right] \phi_{h v} J_{h v}}+\frac{4 F D_{\text {diffusion }}\left[F e^{3+}\right]^{*} r_{T}}{F \pi r_{T}^{2} l\left[\mathrm{BiVO}_{4}^{0}\right] k_{o x}^{\prime}\left[F e^{3+}\right]^{*}} \\
& =\frac{1}{I_{T, \text { cond }}}+\frac{4 D_{\text {diffusion }}\left[F e^{3+}\right]^{*}}{\pi r_{T} l\left[\mathrm{BiVO}_{4}{ }^{0}\right] \phi_{h v} J_{h v}}+\frac{4 D_{\text {diffusion }}}{\pi r_{T} l\left[\mathrm{BiVO}_{4}{ }^{0}\right] k_{o x}^{\prime}}
\end{aligned}
$$

Comparison to uncomplicated first order process at the sample, ${ }^{7,8}$

$$
\begin{aligned}
& \frac{1}{I_{S}}=\frac{1}{I_{T, \text { cond }}}+\frac{4}{\pi} \frac{1}{k}, k_{\text {eff }}=k \frac{D_{\text {diffusion }}}{r_{T}} \\
& \frac{1}{I_{S}}=\frac{1}{I_{T, \text { cond }}}+\frac{4 D_{\text {diffusion }}}{\pi r_{T}} \frac{1}{k_{\text {eff }}} \\
& \frac{1}{I_{S}}=\frac{1}{I_{T, \text { cond }}}+\frac{4 D_{\text {diffusion }}}{\pi r_{T}}\left[\frac{\left[\mathrm{Fe}^{3+}\right]^{*}}{l\left[\mathrm{BiVO}_{4}^{0}\right] \phi_{h v} J_{h v}}+\frac{1}{l\left[\mathrm{BiVO}_{4}^{0}\right] k_{o x}^{\prime}}\right] \\
& k_{\text {eff }}=\frac{l\left[\mathrm{BiVO}_{4}{ }^{0}\right] \phi_{h v} J_{h v} k_{o x}^{\prime}}{k_{o x}^{\prime}\left[\mathrm{Fe}^{3+}\right]^{*}+\phi_{h v} J_{h v}}
\end{aligned}
$$




\section{SI-6 SECM measurements for interfacial reaction kinetics in photoelectrochemical water splitting}

SI-6.1 Normalized approach curves towards FTO/BiVO 4 film under blue illumination and in dark

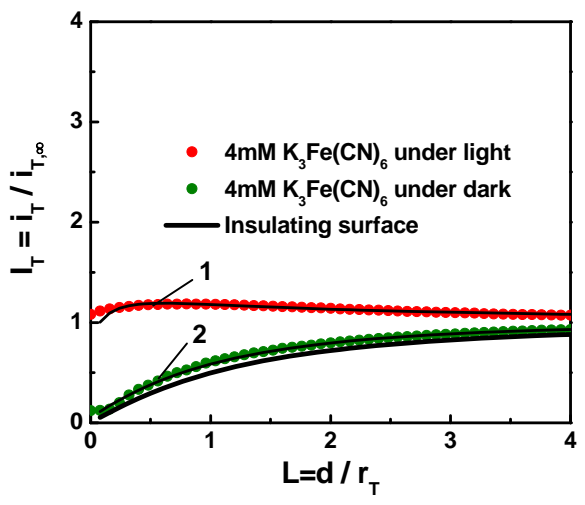

Figure S4. Normalized SECM feedback approach curves for the approach of a Pt ultra-microelectrode towards a $\mathrm{FTO} / \mathrm{BiVO}_{4}$ film under dark and illumination by a blue light LED at a constant intensity $J_{\mathrm{hv}}$ of $22.4 \times 10^{-9} \mathrm{~mol} \mathrm{~cm}^{-2} \mathrm{~s}^{-1}$, with $4 \mathrm{mM}\left[\mathrm{Fe}(\mathrm{CN})_{6}\right]^{3-}$ as redox mediator, scan rate $=1 \mu \mathrm{m} \mathrm{s}^{-1}, \mathrm{E}\left(\mathrm{T},\left[\mathrm{Fe}(\mathrm{CN})_{6}\right]^{3-}\right)=$ $0 \mathrm{~V}$ (vs. $\mathrm{Ag} / \mathrm{AgCl}$ ), $\mathrm{r}_{\mathrm{T}}=12.5 \mu \mathrm{m}$. Solid lines are calculated curves for the approach of a $\mathrm{UME}$ with $\mathrm{RG}=10$ toward an inert insulating surface (line at the bottom), and toward samples with first-order kinetics of mediator recycling using normalized rate constant $\kappa:$ 1) $0.87,2) 0.012$.

a)

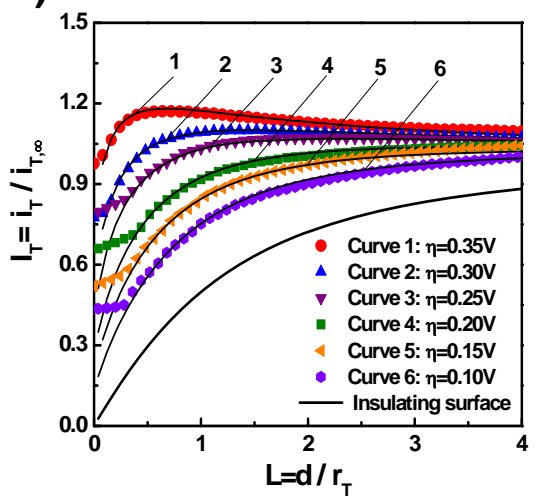

b)

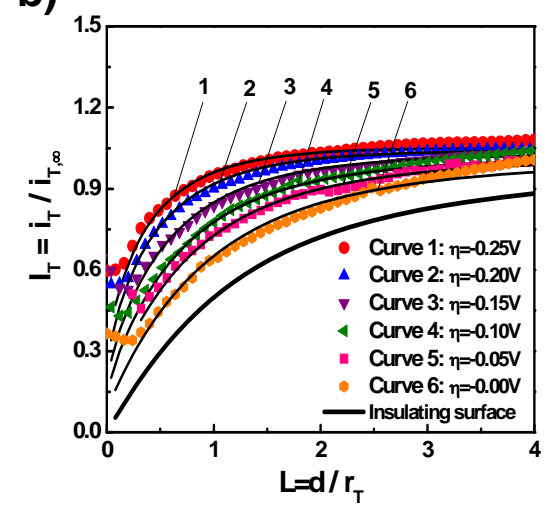

Figure S5. Normalized SECM feedback approach curves for Pt ultra-microelectrode towards FTO/BiVO 4 film under dark: a) $2 \mathrm{mM}\left[\mathrm{Fe}(\mathrm{CN})_{6}\right]^{3-}$ and b) $2 \mathrm{mM}\left[\mathrm{Fe}(\mathrm{CN})_{6}\right]^{4-}$ with various over-potential $\eta\left(=\mathrm{E}_{\mathrm{app}}-\mathrm{E}_{1 / 2}{ }^{0}\right)$, at a scan rate of $1 \mu \mathrm{m} \mathrm{s}^{-1}, \mathrm{E}\left(\mathrm{T},\left[\mathrm{Fe}(\mathrm{CN})_{6}\right]^{3-}\right)=0 \mathrm{~V}, \mathrm{E}\left(\mathrm{T},\left[\mathrm{Fe}(\mathrm{CN})_{6}\right]^{3-}\right)=0.5 \mathrm{~V}(\mathrm{vs} . \mathrm{Ag} / \mathrm{AgCl}), r_{\mathrm{T}}=12.5 \mu \mathrm{m}$.

Solid lines are calculated curves for an approach of an $\mathrm{UME}$ with $\mathrm{RG}=10$ towards an inert insulating 
surface, and the first-order kinetics toward samples using normalized rate constant $\kappa$ : (a) 1) $0.84,2$ ) 0.56 , 3) 0.42 , 4) 0.24 , 5) 0.16 , 6) 0.09 ; (b) 1) 0.29 , 2) 0.24 , 3) 0.16 , 4) $0.11,5) 0.08$, 6) 0.05 .

SI-6.2 Normalized approach curves towards FTO/Mo: $\mathrm{BiVO}_{4}$ film under dark
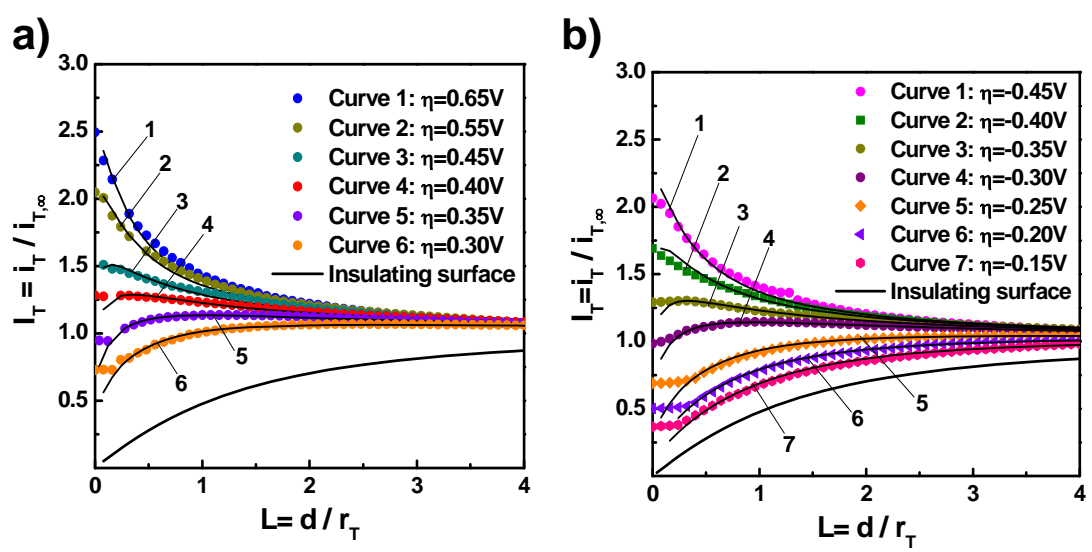

Figure S6. Normalized SECM feedback approach curves for Pt ultra-microelectrode towards FTO/Mo: $\mathrm{BiVO}_{4}$ film under dark: (a) $2 \mathrm{mM}\left[\mathrm{Fe}(\mathrm{CN})_{6}\right]^{3-}$, and (b) $2 \mathrm{mM}\left[\mathrm{Fe}(\mathrm{CN})_{6}\right]^{4-}$ with various over-potential $\eta\left(=\mathrm{E}_{\mathrm{app}}-\mathrm{E}_{1 / 2}{ }^{0}\right)$ for the photo-anode, at a scan rate of $1 \mu \mathrm{m} \mathrm{s}^{-1} . \mathrm{E}\left(\mathrm{T},\left[\mathrm{Fe}(\mathrm{CN})_{6}\right]^{3-}\right)=0 \mathrm{~V}, \mathrm{E}\left(\mathrm{T},\left[\mathrm{Fe}(\mathrm{CN})_{6}\right]^{4-}\right.$ )$=0.5 \mathrm{~V}$ (vs. $\mathrm{Ag} / \mathrm{AgCl}), r_{T}=12.5 \mu \mathrm{m}$. Solid lines are calculated curves for an approach of an UME with $\mathrm{RG}=10$ towards an inert insulating surface, and the first-order kinetics toward samples using normalized rate constant $\kappa$ : (a) 1) 2.90, 2) 2.32, 3) 1.51, 4) 1.09, 5) 0.68, 6) 0.37; (b) 1) 2.50, 2) 1.81, 3) 1.12, 4) 0.71, 5) $0.25,6) 0.12,7) 0.06$. 
SI-6.3 Normalized approach curves towards FTO/Mo: $\mathrm{BiVO}_{4}$ film under blue illumination

a)

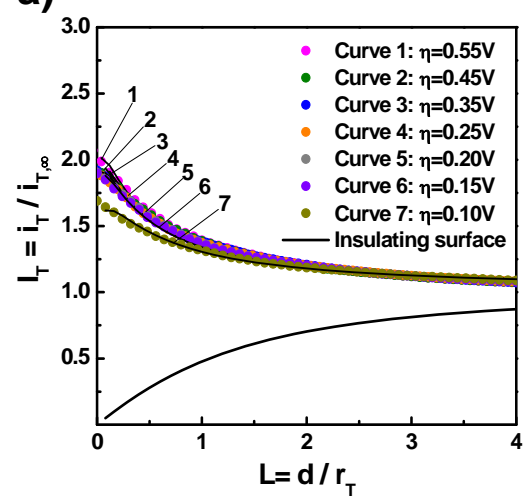

b)

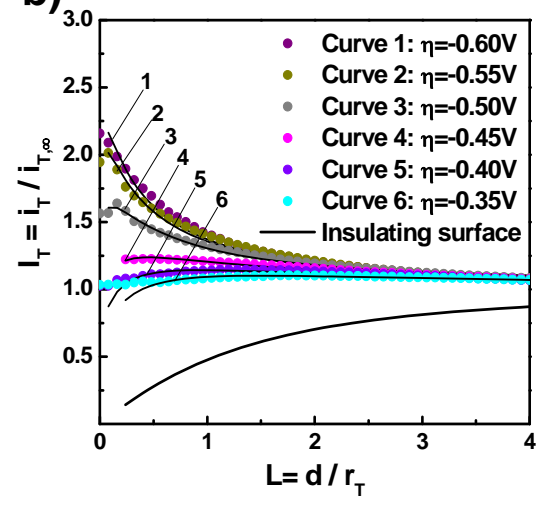

Figure S7. Normalized SECM feedback approach curves for Pt ultra-microelectrode towards FTO/Mo:

$\mathrm{BiVO}_{4}$ film under illumination by a blue light LED: (a) $2 \mathrm{mM}\left[\mathrm{Fe}(\mathrm{CN})_{6}\right]^{3-}$, and (b) $2 \mathrm{mM}\left[\mathrm{Fe}(\mathrm{CN})_{6}\right]^{4-}$ with various over-potential $\eta\left(=\mathrm{E}_{\mathrm{app}}-\mathrm{E}_{1 / 2}{ }^{0^{\prime}}\right)$ for the photo-anode, at a scan rate of $1 \mu \mathrm{m} \mathrm{s}^{-1} . \mathrm{E}\left(\mathrm{T},\left[\mathrm{Fe}(\mathrm{CN})_{6}\right]^{3-}\right)=0 \mathrm{~V}$, $\mathrm{E}\left(\mathrm{T},\left[\mathrm{Fe}(\mathrm{CN})_{6}\right]^{4-}\right)=0.5 \mathrm{~V}$ (vs. $\left.\mathrm{Ag} / \mathrm{AgCl}\right), r_{T}=12.5 \mu \mathrm{m}$. Solid lines are calculated curves for an approach of an $\mathrm{UME}$ with $\mathrm{RG}=10$ towards an inert insulating surface, and the first-order kinetics toward samples using normalized rate constant $\kappa$ : (a) 1) 2.29, 2) 2.20, 3) 2.18, 4) 2.14, 5) 2.12, 6) 2.09, 7) 2.05; (b) 1) 2.56, 2) $2.31,3) 1.68$, 4) $0.97,5) 0.72,6) 0.56$.

SI-6.4 Calculated data from SECM measurement

Table S1. Full data sets of Figure 3b

\begin{tabular}{|c|c|c|}
\hline$[\mathrm{C}] / 10^{-6} \mathrm{~mol} \mathrm{~cm}^{-3}$ & $\kappa$ & $k_{\mathrm{eff}} / 10^{-3} \mathrm{~cm} \mathrm{~s}^{-1}$ \\
\hline 0.3 & 5.50 & 33.44 \\
\hline 0.6 & 2.33 & 14.22 \\
\hline 1.0 & 1.35 & 8.21 \\
\hline 2.0 & 0.98 & 0.60 \\
\hline 4.0 & 0.87 & 0.40 \\
\hline
\end{tabular}


Table S2. Full data sets of Figure 4a

\begin{tabular}{|c|c|c|c|}
\hline$\eta / V$ & $\kappa$ & $k_{\text {eff }} / 10^{-3} \mathrm{~cm} \mathrm{~s}^{-1}$ & In $\left(k_{\text {eff }}\right)$ \\
\hline 0.10 & 0.91 & 5.53 & -5.19 \\
\hline 0.15 & 0.95 & 5.78 & -5.15 \\
\hline 0.20 & 1.04 & 6.32 & -5.06 \\
\hline 0.25 & 1.12 & 6.81 & -4.98 \\
\hline 0.30 & 1.23 & 7.48 & -4.89 \\
\hline 0.35 & 1.32 & 8.03 & -4.82 \\
\hline 0.40 & 1.48 & 9.00 & -4.71 \\
\hline 0.45 & 1.59 & 9.67 & -4.67 \\
\hline
\end{tabular}

Table S3. Full data sets of Figure 4b

\begin{tabular}{|c|c|c|c|}
\hline$\eta / \mathrm{V}$ & $\kappa$ & $k_{\mathrm{eff}} / 10^{-3} \mathrm{~cm} \mathrm{~s}^{-1}$ & In $\left(k_{\mathrm{eff}}\right)$ \\
\hline-0.40 & 4.01 & 21.20 & -3.85 \\
\hline-0.35 & 2.25 & 11.90 & -4.42 \\
\hline-0.30 & 1.72 & 9.10 & -4.69 \\
\hline-0.25 & 1.25 & 6.61 & -5.02 \\
\hline-0.20 & 1.14 & 6.03 & -5.11 \\
\hline
\end{tabular}


Table S4. Full data sets of Figure S4a

\begin{tabular}{|c|c|c|c|}
\hline$\eta / \mathrm{V}$ & $\kappa$ & $k_{\text {eff }} / 10^{-3} \mathrm{~cm} \mathrm{~s}^{-1}$ & In $\left(k_{\text {eff }}\right)$ \\
\hline 0.10 & 0.09 & 0.55 & -7.47 \\
\hline 0.15 & 0.16 & 0.97 & -6.93 \\
\hline 0.20 & 0.24 & 1.46 & -6.52 \\
\hline 0.25 & 0.42 & 2.55 & -5.99 \\
\hline 0.30 & 0.56 & 3.40 & -5.68 \\
\hline 0.35 & 0.84 & 5.11 & -5.28 \\
\hline
\end{tabular}

Table S5. Full data sets of Figure S4b

\begin{tabular}{|l|c|c|c|}
\hline$\eta / \mathrm{V}$ & $\kappa$ & $\begin{array}{c}k_{\text {eff }} / 10^{-3} \mathrm{~cm} \mathrm{~s}^{-} \\
1\end{array}$ & In $\left(k_{\text {eff }}\right)$ \\
\hline-0.25 & 0.29 & 1.53 & -6.47 \\
\hline-0.20 & 0.24 & 1.27 & -6.69 \\
\hline-0.15 & 0.16 & 0.85 & -7.05 \\
\hline-0.10 & 0.11 & 0.58 & -7.46 \\
\hline-0.05 & 0.08 & 0.42 & -7.78 \\
\hline-0.00 & 0.05 & 0.26 & -8.17 \\
\hline
\end{tabular}


Table S6. Full data sets of Figure S5a

\begin{tabular}{|c|c|c|c|}
\hline$\eta / \mathrm{V}$ & $\kappa$ & $k_{\text {eff }} / 10^{-3} \mathrm{~cm} \mathrm{~s}^{-1}$ & In $\left(k_{\text {eff }}\right)$ \\
\hline 0.30 & 0.37 & 4.14 & -5.48 \\
\hline 0.35 & 0.68 & 5.06 & -5.29 \\
\hline 0.40 & 1.09 & 6.61 & -5.02 \\
\hline 0.45 & 1.51 & 9.17 & -4.69 \\
\hline 0.55 & 2.32 & 14.10 & -4.26 \\
\hline 0.65 & 2.9 & 17.65 & -4.03 \\
\hline
\end{tabular}

Table S7. Full data sets of Figure S5b

\begin{tabular}{|c|c|c|c|}
\hline$\eta / \mathrm{V}$ & $\kappa$ & $k_{\mathrm{eff}} / 10^{-3} \mathrm{~cm} \mathrm{~s}^{-1}$ & In $\left(k_{\mathrm{eff}}\right)$ \\
\hline-0.45 & 2.50 & 13.26 & -4.32 \\
\hline-0.40 & 1.81 & 9.55 & -4.65 \\
\hline-0.35 & 1.12 & 5.91 & -5.13 \\
\hline-0.30 & 0.71 & 3.76 & -5.58 \\
\hline-0.25 & 0.25 & 1.33 & -6.62 \\
\hline-0.20 & 0.12 & 0.63 & -7.38 \\
\hline-0.15 & 0.06 & 0.32 & -8.03 \\
\hline
\end{tabular}


Table S8. Full data sets of Figure S6a

\begin{tabular}{|c|c|c|c|}
\hline$\eta / \mathrm{V}$ & $\kappa$ & $k_{\text {eff }} / 10^{-3} \mathrm{~cm} \mathrm{~s}^{-1}$ & In $\left(k_{\text {eff }}\right)$ \\
\hline 0.10 & 2.05 & 12.49 & -4.38 \\
\hline 0.15 & 2.09 & 12.74 & -4.36 \\
\hline 0.20 & 2.12 & 12.94 & -4.35 \\
\hline 0.25 & 2.14 & 13.03 & -4.34 \\
\hline 0.35 & 2.18 & 13.28 & -4.32 \\
\hline 0.45 & 2.20 & 13.40 & -4.31 \\
\hline 0.55 & 2.29 & 13.91 & -4.28 \\
\hline
\end{tabular}

Table S9. Full data sets of Figure S6b

\begin{tabular}{|c|c|c|c|}
\hline$\eta / \mathrm{V}$ & $\kappa$ & $k_{\mathrm{eff}} / 10^{-3} \mathrm{~cm} \mathrm{~s}^{-1}$ & In $\left(k_{\text {eff }}\right)$ \\
\hline-0.60 & 2.56 & 13.55 & -4.31 \\
\hline-0.55 & 2.31 & 12.22 & -4.41 \\
\hline-0.50 & 1.68 & 8.87 & -4.72 \\
\hline-0.45 & 0.97 & 5.12 & -5.27 \\
\hline-0.40 & 0.72 & 3.78 & -5.57 \\
\hline-0.35 & 0.56 & 2.96 & -5.83 \\
\hline
\end{tabular}



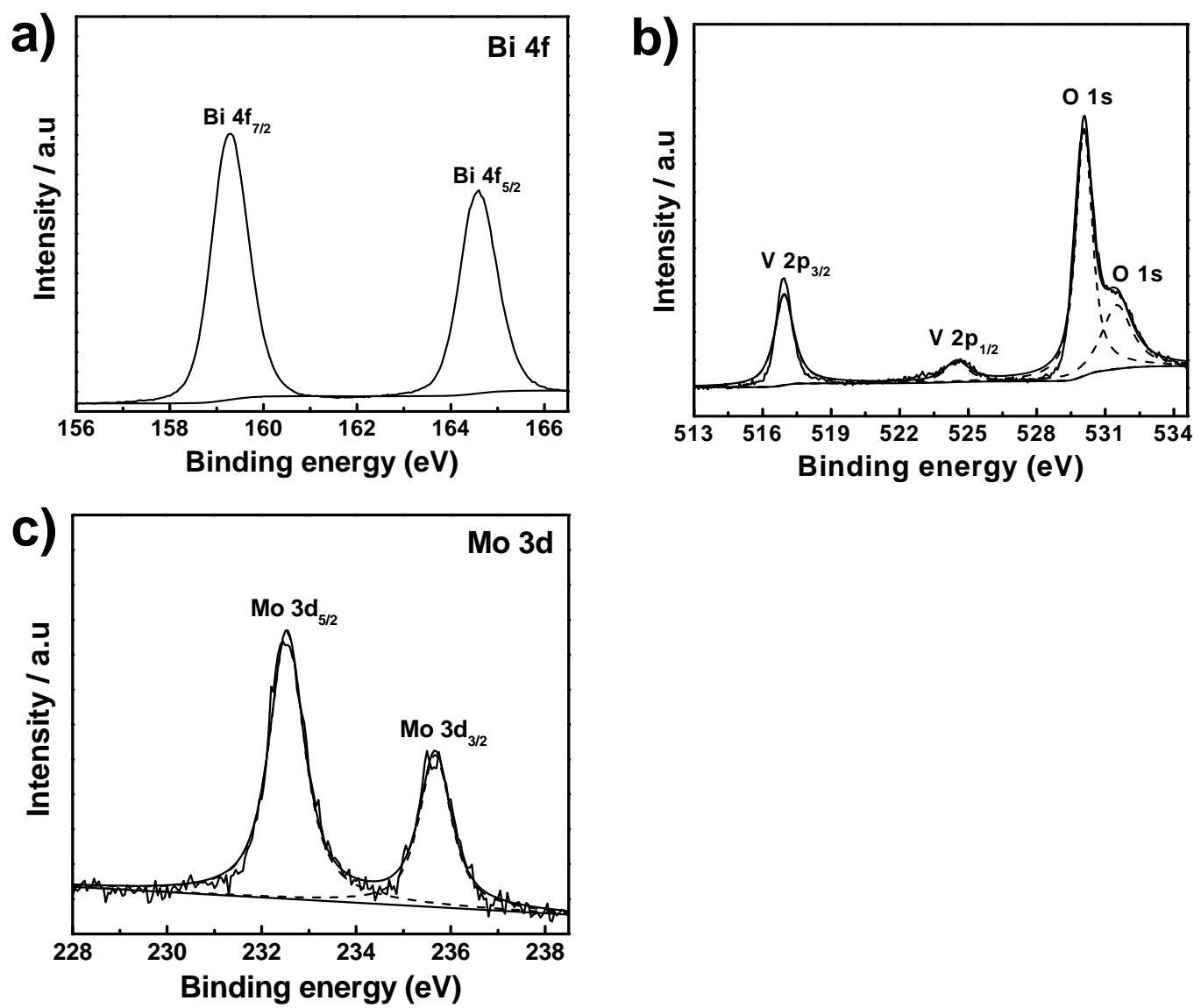

Figure S8. XPS spectra data for the sample Mo: $\mathrm{BiVO}_{4}$ : (a) $\mathrm{Bi}^{3+}$, (b) $\mathrm{V}^{5+}$ and $\mathrm{O}^{2-}$, (c) $\mathrm{Mo}^{6+}$ regions, the binding energies are calibrated by the binding energy of C $1 \mathrm{~s} 284.8 \mathrm{eV}$.

\section{References}

(1) McDonald, K.; Choi, K. A New Electrochemical Synthesis Route for A BiOI Electrode and Its Conversion to A Highly Efficient Porous $\mathrm{BiVO}_{4}$ Photoanode for Solar Water Oxidation. Energy Environ. Sci. 2012, 5, 8553-8557.

(2) Kim, T.; Choi, K. Nanoporous $\mathrm{BiVO}_{4}$ Photoanodes with Dual-Layer Oxygen Evolution Catalysts for Solar Water Splitting. Science 2014, 343, 990-994.

(3) Bard, A. J.; Mirkin, M. V. Scanning Electrochemical Microscopy, 2nd ed., CRC, New York, 2012. 
(4) Cornut, R.; Lefrou, C. New Analytical Approximation of Feedback Approach Curves with A Microdisk SECM Tip and Irreversible Kinetic Reaction at the Substrate. J. Electroanal. Chem. 2008, 621, 178184.

(5) Mengesha, T.; Nonomura, K.; Vlachopoulos, N.; Hagfeldt, A.; Wittstock, G. Effect of Cation on Dye Regeneration Kinetics of N719-Sensitized $\mathrm{TiO}_{2}$ Films in Acetonitrile-Based and Ionic-Liquid-Based Electrolytes Investigated by Scanning Electrochemical Microscopy. J. Phys. Chem. C 2012, 116, 43164323.

(6) Rust, F.; Vaughan, W. Oxidation of Hydrocarbons Catalyzed by Hydrogen Bromide-Summary. Ind. Eng. Chem. 1949, 41, 2595-2597.

(7) Wei, C.; Bard, A. J.; Mirkin, M. V. Scanning Electrochemical Microscopy. 31. Application of SECM to the Study of Charge Transfer Processes at the Liquid/Liquid Interface. J. Phys. Chem. 1995, 99, 16033-16042.

(8) Lefrou, C.; Cornut, R. Analytical Expressions for Quantitative Scanning Electrochemical Microscopy (SECM). ChemPhysChem, 2010, 11, 547-556. 